\title{
Research and methodology in healthcare interpreting
}

\author{
Franz Pöchhacker \\ Center for Translation Studies, University of Vienna
}

This paper reviews the evolution and main strands of research on interpreting in health care, one of the most important domains of interpreting in the community. The diverse body of literature generated since about the 1970s is first described with regard to different disciplinary perspectives and then surveyed in terms of thematic orientations, including a focus on the interpreting product, interpreter performance, communicative practices, and the provision of care. A number of examples are cited to illustrate both the breadth of content and the variety of methods used. The latter are then discussed from an overall perspective with regard to some broad methodological distinctions and choices.

\section{Introduction}

Healthcare settings constitute one of the most important domains of community-based interpreting. It was in the field of health care that some of the earliest initiatives for the systematic provision of community interpreting services were taken, in the 1970s, and it was medical interpreters who, two decades later, formed the first specialized professional bodies for community-based interpreting outside the judicial domain. Not surprisingly, this extensive field of practice has attracted considerable research interest, though not necessarily among scholars in interpreting studies, or translation studies in general. Biased toward the 'high end' of the profession, interpreting researchers, typically affiliated with university-level institutions for conference interpreter training, had little reason to take an interest in the phenomenon.

Approaching the activity of interpreting in health care from entirely different disciplinary paradigms, it was researchers in the fields of language and communication, and in the health and social sciences who initiated empirical research on healthcare interpreting several decades before the topic received international attention at the first Critical Link conference in 1995. Ever since that landmark event, efforts to promote this field of practice and research have intensified considerably, amply justifying the present attempt at taking stock of what has been done so far. As indicated in the (somewhat misleadingly additive) title, my intention in this paper is to offer a broad overview of research in the field of healthcare interpreting, with special consideration for methodological issues.

The first step toward the goal of an analytical survey of healthcare interpreting research must be to recognize, and make sense of, its diversity. There is not only one literature to be surveyed, but various literatures, 
generated by researchers in a number of different disciplines. Moreover, publications in this area include many descriptive contributions and discussions on issues of professionalization such as training, service management and professional ethics, which are essential to progress in the field but would not necessarily be regarded as (empirical) research. Even on this narrower understanding of research, the body of literature under review $^{1}-$ or rather, the selection of publications known and available to $\mathrm{me}^{2}$

- is highly heterogeneous. I will attempt to group it into five thematic orientations or themes, citing examples to illustrate both the breadth of content and the variety of methods used. The latter will be discussed with regard to some broad methodological distinctions and basic choices in inquiry, with a few concluding thoughts on dominant themes and policy issues for further research.

\section{Disciplines}

By its very nature, the topic of interpreting in health care will be looked at from at least two points of view - that of interpreting and that of the health sciences. In actual fact, the number of disciplinary perspectives on this phenomenon is much larger. The 'health sciences' are obviously not a single academic discipline but an agglomeration of numerous fields (like primary care, nursing, emergency medicine, pediatrics, psychiatry, psychotherapy) and their often interdisciplinary extensions, such as medical anthropology, medical communication, medical ethics and medical sociology, not to mention the focus on transcultural issues in any of these fields, as in transcultural psychiatry, transcultural nursing or transcultural pediatrics.

Some of these fields have emerged only recently, as is also the case with interpreting studies as a (sub)discipline within the wider field of translation studies. Indeed, when interpreting in healthcare settings first became an object of inquiry, in the 1970s, interpreting as a field of academic study in its own right hardly existed. The earliest contributions to the literature on healthcare interpreting thus came from the fields of nursing, social work, linguistics, and psychiatry. Most of these domains continued to fuel the development of research, consistently or at different times, while new branches of the health sciences and of the communication-oriented social sciences gradually joined in. In this first sketch of research and its evolution since the 1970s, the following subsections present examples of work done from various disciplinary perspectives, using authors' professional and academic affiliations and the medium of publication as rough-and-ready categorization criteria. The order of presentation to some extent reflects the field's chronological evolution over some three decades, and more detailed attention will be given in this section to some of the pioneering studies, whereas newer research will be covered more extensively in section 2 . 


\subsection{Nursing}

Appropriately, given the significant and possibly undervalued contribution of nursing to effective medical care, the first paper on record to address the use of interpreters was published in a nursing journal. Richie (1964) discussed qualities desirable in a medical interpreter, such as respect for confidentiality and a thorough knowledge of the patient's culture. Stressing also the need to avoid personal involvement, the author discouraged the use of family members, particularly children, as interpreters. While the literature on transcultural nursing, largely associated with the work of Leininger (e.g. 1991), generally fails to engage with the issue of interpreter use, research from the perspective of nursing science has gained ground in recent years, not least thanks to the work of Alexander Bischoff and associates in Switzerland (cf. Bischoff, in this volume).

\subsection{Linguistics}

The first linguistic study of medical interpreting was carried out by Ranier Lang (1975), a linguist in the Research School of Pacific and Asian Studies of the Australian National University. On the basis of mediated doctorpatient interactions tape-recorded in two Papua New Guinean hospitals in the late 1960s, Lang analyzed the performance of medical orderlies serving as interpreters between Enga and Tok Pisin. Various reasons for the presence of additions and omissions are discussed, including carelessness, cognitive and linguistic challenges, and "wilful distortion", often as a result of the orderly's dual role as co-worker and interpreter. Few linguists took a similarly profound interest in interpreting in subsequent years, a notable exception being Prince (1986), who analyzed the distribution and accuracy of question-answer exchanges in twelve audiotaped medical interviews with or without (non-professional) interpreters for Spanish-speaking patients. In the seven interpreter-mediated encounters, Prince identified seven types of communication problems, three of which were attributed to interpreter behaviors: answering instead of translating questions, translating incompletely or translating incorrectly.

In a dissertation at Stanford University, Brad Davidson (1998), drawing on a much larger corpus of recorded medical interviews in a Californian hospital, compared ten English-English and ten SpanishEnglish interpreter-mediated encounters and found that the hospital-based (professional) interpreters often assumed the role of "pre-diagnostician", editing or omitting patients' contributions so as to "keep the patient 'on track', and keep the interview moving quickly" (1998: v). On a smaller scale, using two audio-recorded interpreter-mediated consultations between English-speaking doctors and Russian-speaking patients, Bolden (2000) confirmed the finding that interpreters (on staff, with some training in interpreting) get actively involved in the process of history-taking. 
Outside the US, large-scale linguistics-based research on interpreting in hospitals has been carried out particularly at the University of Hamburg Research Centre on Multilingualism (e.g. Meyer 2002). Working in the discourse-analytical framework of functional pragmatics, these researchers have analyzed the performance of lay interpreters (family members and bilingual hospital staff) for Portuguese- and Turkish-speaking patients, with special emphasis on briefings for informed consent (see also Meyer et al. 2003).

\subsection{Mental health}

The most significant driving force in the early development of research on healthcare interpreting was psychiatry. One of the first publications on interpreter use in interviewing, by Bloom et al. (1966), was featured in the journal Mental Hygiene, even though it was derived from a social-work rather than a mental-health project. With reference to two illustrative vignettes, the authors suggested three paradigmatic roles for the interpreter in relation to the interviewer: the interpreter may become the interviewer, serve the latter as a mere tool, or work in partnership with the specialist.

As in the case of nursing and linguistics, some of the earliest work on interpreting in psychiatric practice involved 'remote' cultures, at least from a Western point of view. Price (1975) reported a quantitative analysis of nine doctor-interpreter pairs (3 doctors x 3 interpreters) who saw a number of Hindustani-speaking patients in a Fijian hospital, many of whom were acutely psychotic. Two of the interpreters were orderlies with some interpreting experience, and one was a paranoid schizophrenic in remission, with no experience as an interpreter but a better educational background. Assessing both the error rate on hundreds of questions and the interpreters' linguistic proficiency, Price found a significantly higher percentage of errors (omissions, distorted questions, additions, etc.) in the performances of the two orderlies, as well as an effect of directionality: patient-topsychiatrist interpreting was significantly more error-prone than the reverse process. Aside from a high rate of omissions in relaying patients' answers to the psychiatrist, the author also found clinically significant additions, e.g. in a patient's description of hallucinatory voices, and emphasized that "an interpreter's apparent competence may readily be mistaken for true competence" (1975: 263).

In the US, pioneering work on interpreting in the psychopathological evaluation of non-English-speaking patients was done by Luis Marcos and associates in New York City. Marcos (1979) first conducted discussions to elicit the views of eight English-speaking psychiatrists and six bilingual hospital employees with extensive interpreting experience, and subsequently audiotaped eight interpreter-mediated psychiatric evaluations of Spanishand Chinese-speaking patients for content analysis. Half of the interviews were interpreted by a bilingual psychiatric nurse, two by a nurse's aid with no background in psychiatry, and two by patients' relatives. Comparative 
assessment of the interpreted material for "clinical equivalence" revealed "consistent, clinically relevant, interpreter-related distortions", associated with interpreters' lack of language competence, translation skills and specialized knowledge as well as the adoption of third-party "attitudes", particularly in patients' relatives (1979: 173). In a similar context, Vasquez \& Javier (1991) described two case examples, one of them with an excerpt from the error-ridden interview, to caution against the use of untrained interpreters and "highlight the deleterious effect of the errors an interpreter can make" (1991: 165). The authors listed five basic categories of error: omission, addition, condensation, substitution, and role exchange.

In the course of the 1990s, a growing body of literature on the use of interpreters in psychiatry and psychotherapy emerged, in the US as well as in other countries. Westermeyer (1990), using a number of illustrative cases, discussed the skills required of interpreters in psychiatric assessment and treatment, and described three conceptual role models reminiscent of those already found in Bloom et al. (1966): the triangle model (involving transference, countertransference and a co-worker relationship); the black box model ("the interpreter as a word unscrambler"); and the bilingual worker model (the interpreter as a "junior clinician"). Work along these lines has also been reported, for example, from Germany (Holzmann et al. 1994) and South Africa (Drennan \& Swartz 1999). Focusing on interpreter use in psychotherapy, some authors have reported more extensive descriptions of individual cases (e.g. Baxter \& Cheng 1996; Leyer 1990 and Porter 1999), while others, such as Hanneke Bot (2005a, 2005b), have also put the emphasis on patterns of discourse. A special domain in this regard is interpreting for victims of torture (e.g. Tribe 1999) which, regrettably, is likely to require continued research attention in the future.

\subsection{Medical sciences}

In primary care and general medicine the issue of language barriers and overcoming them by using interpreters came to the fore in the course of the 1980s, preceded by pioneering work in expatriate contexts: Launer (1978) briefly reported on interpreter use by English-speaking doctors in a Nigerian outpatient department. Analyzing recorded consultations with 30 Hausa patients in which seven medical orderlies served as interpreters, Launer found deviations from the standard of accurate and complete ("word-for-word") translation and noted that "interpreters were inclined to conduct much of the consultations themselves" (1978: 934). Acknowledging the similar findings of Lang (1975), Launer concluded that the problem was not a local one but had to do with the nature of medical interviewing and the demands of interpreting. For the latter, he suggested that interpreters should have "training in language and the rudiments of interpretation" and that "doctors should be taught how to use them" (1978: 935). 
A comprehensive discussion of the role of interpreters and the broader issues of cross-cultural health care by Putsch (1985), published in the Journal of the American Medical Association, could be said to have put the topic of medical interpreting on the mainstream agenda. No less influential was the review paper by Woloshin et al. (1995) on "language barriers in medicine", published ten years later in the same journal. Citing more than 60 papers and documents, the authors advocate the use of professional interpreters as "the standard solution to language barriers" (1995: 724) and discuss the clinical as well as legal risks posed by suboptimal solutions such as the use of "family, friends and ad hoc interpreters" (bilingual employees or other patients). One of the studies cited as evidence was the analysis of Ebden et al. (1988) of four videotaped consultations with Gujarati-speaking patients in which family members served as interpreters. Out of 143 questions of different complexity (simple, complex or serial), between a quarter and half of the doctor's interview questions were mistranslated or omitted, and more than 80 words and phrases (anatomical terms, symptoms) were mistranslated or omitted by at least one interpreter. Significantly, the interviews appeared "reasonably normal" to the doctors, even though they were all "badly misleading for linguistic or cultural reasons" and "would have made it difficult to form a correct initial diagnosis" (Ebden et al. 1988: 347).

In the course of the 1990s, efforts to address the issue of language barriers in research and practice intensified, and medical researchers went beyond the focus on interpreting as such to study the various implications of patients' limited language proficiency for access to services and the quality of care. The effect of language barriers on the uptake of preventive services (e.g. in gynecology and HIV risk reduction), on treatment and compliance with prescriptions, and on clinical outcomes, patient satisfaction and medical follow-up, especially in the emergency department (e.g. Baker et al. 1998 and Bernstein et al. 2002), have been examined in a number of studies (cf. section 2.4), as documented in the annotated bibliography of medical research on language barriers in health care compiled by Jacobs et al. (2003).

\subsection{Social sciences}

In contrast to medical research seeking to quantify clinical outcomes and assess the impact of language barriers on the delivery of care, researchers approaching medical communication from the perspective of cultural anthropology have foregrounded the role of culture in the patient-provider relationship. While Putsch (1985: 3344) certainly drew attention to "the complexities of cross-cultural communication", the focus on ethnomedical considerations in healthcare interpreting, particularly in relation to indigenous languages and cultures, is most closely associated with the work of Joseph Kaufert and associates at the University of Manitoba (e.g. Kaufert $\&$ Koolage 1984). Drawing on the work of 1970s advocates of an 
alternative to the biomedical model (e.g. Kleinman et al. 1978), medical anthropologists have pointed out how patients' cultural beliefs and value systems about illness - as opposed to healthcare professionals' biomedical notion of disease - confront the healthcare interpreter with major ethical challenges and role conflicts (e.g. Kaufert \& Koolage 1984 and Kaufert \& Putsch 1997).

But social-science approaches to communication with patients from other cultures have not invariably adopted the anthropological or ethnomedical perspective. Though sociologists have made vital contributions to the study of communication in health care (e.g. Fisher \& Todd 1983), most research on doctor-patient interaction has concentrated on socio-psychological factors and tended to ignore the intercultural dimension (e.g. Ong et al. 1995). Aaron Cicourel (1981), for instance, in a review paper on "language and medicine", discusses a consultation between an English-speaking physician and a Spanish-speaking family in which a relative acts as interpreter. While noting the "general confusions and misunderstandings that occurred frequently throughout the interview" and pointing to contemporary media reports on communication barriers in medical care, Cicourel (1981: 425f.) limits his comments on the impact of interpreting on the interaction to the statement that "some of the communication gets a little garbled in the translation."

Nevertheless, sociological and sociolinguistic approaches to the study of interpersonal interaction have been essential to the emergence of discourse-based research on communication (and interpreting) in health care - an inherently interdisciplinary domain, in which it seems impossible to distinguish between linguists, sociolinguists, discourse analysts, communication theorists, and medical communication researchers. Mindful of this disciplinary heterogeneity, I will use the term 'communication studies' as a broad label subsuming various research approaches to medical communication.

\subsection{Communication studies}

Communication has become a major area of specialization within medicine over the past two decades, focusing on such topics as interaction analysis, teaching and assessing communicative skills in medical students and clinicians, counseling for various patient groups (cancer patients, the elderly, etc.) and patient education. So far, communicating with patients from other cultures, let alone interpreter use, has not figured prominently in the conferences ${ }^{3}$ and publications devoted to this field. Nor has communication research, as undertaken from sociolinguistic and discourse analytical perspectives, including conversation analysis and critical discourse analysis (cf. Sarangi 2004: 1). One might indeed discern a broadly dual development of research on communication in health care: one with a 'linguistic' (discourse analytical) orientation, which would thus come under the heading of section 1.2 above, and another with an inherently medical outlook, 
championed by researchers within the medical sciences. To date, there seems to be little interaction between these two communication-oriented communities, neither of which has yet shown particular concern for healthcare communication mediated by interpreters.

This also applies, generally speaking, to communication studies as such, that is, as an academic discipline. Nevertheless, researchers with this affiliation have contributed some of the more recent studies on the key issue of medical interpreters' role performance. Hsieh (2006) examined role conflicts on the basis of in-depth interviews with 26 experienced medical interpreters for 17 languages in the midwestern US. She identified four sources of conflicts, including participant dynamics, institutional constraints, and unrealistic expectations, as well as various strategies for resolving them. Essentially, she finds that the behavior of the other participants in the interaction influences the interpreter's communicative strategies and role management, often requiring a departure from the "conduit role". With regard to the latter, Hsieh (2006) refers to DysartGale's (2005) discussion of interpreting practice in the light of communication-theoretical models, and to her conclusion (based on interviews with medical interpreters) that "the conduit model provides neither a complete description of interpreter work in clinical settings nor adequate grounding for ethical decision making in interpreter practice" (Dysart-Gale 2005: 98). Neither of these authors makes any reference to the work of Wadensjö (1998), their fellow communication scholar who, more than a decade earlier, had rejected the channel metaphor and highlighted the interpreter's intertwined functions of translating and 'coordinating others' talk". From an interpreting studies perspective, in contrast, Cecilia Wadensjö is acknowledged for her leading role in the emergence of the dialogic discourse-based paradigm of interpreting research, which is why it may be considered appropriate to include her in the section below.

\subsection{Interpreting studies}

As a newly emerged (sub)discipline within the wider field of translation studies, interpreting studies has only recently begun to contribute to the literature on interpreting in health care. While most members of this community are professional interpreters, interpreter educators, graduate students and interpreting researchers (often combining two or more of these roles), the field has varied disciplinary roots, including cognitive psychology, applied linguistics, and language and communication studies. It is from the latter that Cecilia Wadensjö, who was trained as a dialogue interpreter, emerged in the early 1990s. Working with discourse scholar Per Linell, she analyzed a corpus of audio-recorded interpreter-mediated encounters, 13 of which involved nurses or doctors in healthcare or childcare clinics in Sweden. Her discourse-analytical research, which draws on Bakhtin's dialogic theory of language and communication as well as Goffman's sociology of interaction, was among the earliest to give an authoritative 
voice to the community interpreter. In the highly developed world of medical research, that voice had hardly been heard, and with few exceptions (e.g. Haffner 1992), interpreters had been only objects of study rather than protagonists of research on their professional domain.

Other researchers with an immediate involvement in healthcare interpreting include Jan Cambridge (1999), Brett Rosenberg (2002) and particularly Claudia Angelelli (2003, 2004a, 2004b, 2006), who has studied medical interpreters from a psychometric as well as an ethnographic perspective. Various contributions have also been made by researchers with a background in other areas of interpreting studies (e.g. Dubslaff \& Martinsen 2005; Merlini \& Favaron 2005; Pöchhacker 2000a, 2000b; Pöchhacker \& Kadric 1999 and Tomassini \& Nicolini 2005). Given the inherent interdisciplinarity of healthcare interpreting as an object of study, and of interpreting studies as an amalgam of socio-psycho-linguistic approaches, the criteria used here - authors' affiliation and medium of publication - are not always sufficient to decide who and what should be classified as research(ers) pertaining to this discipline. Nevertheless, journals such as Interpreting, which devoted a special issue to healthcare interpreting in 2005, and the proceedings volumes of the Critical Link conferences (Carr et al. 1997; Roberts et al. 2000; Brunette et al. 2003 and Englund Dimitrova et al. 2006) can serve as a point of reference.

\section{Themes}

Classifying research by disciplinary background, though useful in tracing the origins and development of the field, is not sufficient to sketch an overall picture of the state of art. In the present section I will therefore attempt to group research on healthcare interpreting by thematic orientation.

Most broadly, one might distinguish between studies on the problems and those on solutions. The former are typically concerned with identifying language as a barrier to health care and assessing the need for mediated communication. Solution-oriented research, on the other hand, would investigate the nature and impact of interpreter use in health care, with particular regard to clinical outcomes, quality of care, and economic implications. A focus on the 'problem', however, quickly reveals that language barriers are only one aspect of the broader concern with 'culturally and linguistically appropriate services' (CLAS) in health care, and that the use of interpreters is only one solution. Research in the medical and social sciences (cf. section 1.4), particularly in public health, rightly addresses this bigger picture, and broader theme. Nevertheless, for the purpose of this review, the focus will be on interpreting, for which I see five thematic orientations in research to date - mnemonically labeled as product, performance, practices, provision and policy. These will be illustrated below with reference to selected studies and attention to methodological details as well as content and findings. 


\subsection{Interpreting product}

Ever since the earliest studies on interpreting in health care, researchers have examined the interpreter's linguistic output, or product, for instances of mistranslation, usually classified as omissions, additions or distortions, as used also in studies of simultaneous interpretations at the time. Indeed, Lang (cf. section 1.2) stated that his interpreting data were "judged by standards of behaviour as it is taught by interpreter training schools in Europe" (1975: 173). What is more, he held an impressively sophisticated view of translational accuracy that is well worth quoting in full:

A perfect interpretation contains nothing less than what is necessary to render the original in the stylistically and culturally most appropriate manner in the target language. In its extreme, this would mean no interpretation for a situation where it would be culturally appropriate to say nothing and to 'interpret' where nothing has been said in the original. (Lang 1975: 174)

Using audio-recorded data did not permit Lang (1975) to push his analysis of "translational adequacy" to such extremes, and he limited himself to a description of various types of mistranslation without any quantification, as did Launer (1978). In contrast, Price (cf. section 1.3) calculated error rates based on at least 100 tape-recorded questions and answers per doctorinterpreter pair, an error being "defined as an alteration in meaning rather than as a failure to translate word for word" (1975: 263). Errors were assessed "at playback" by the three doctors themselves, two of which spoke both languages used in the project while the other was assisted by a bilingual nurse. Price also reported a quantification of error types, but without describing the assessment procedure used. It is not clear how one would consistently distinguish between "mistranslation due to carelessness" and "mistranslation due to ignorance of English", or between a "partial omission" and a "faulty summary" by the interpreter (1975: 265).

Such lack of explicitness regarding the method of verbal data analysis also afflicts the study by Ebden et al. (1988), who report percentages of mistranslated questions without explaining how they distinguished between a mistranslation and a legitimate paraphrase. They also quantified the number of mistranslated words or phrases without describing how and by whom the translations were assessed, except for stating that the Gujarati portions of the interviews were "independently translated into English", which points to a comparative assessment based on back-translation. Significantly, the video-recorded interview for each of the four previously seen patients was "conducted as if this was their first visit", which suggests that the study had features of a simulation.

Considerably more methodological rigor and authenticity can be attested to the study by Flores et al. (2003). As part of a larger study 
involving 153 audiotaped visits to a pediatric outpatient clinic, the authors analyzed 13 Spanish-English encounters mediated by the hospital's professional (paid) interpreters or by ad hoc interpreters (nurses, a social worker and an 11-year-old sibling). It is noteworthy that the transcripts (some 50,000 words) were reviewed for accuracy by three different bilingual experts. Errors were assigned to five classes of "misinterpretation", using Barik's main categories of omissions, additions and substitutions complemented by "editorialization" and "false fluency", while excluding "deviations from word-for-word interpretation" that were "attributable to jargon, idioms, or contextual clarifications" (Flores et al. 2003: 7). All errors were also assessed for their "potential clinical consequences". Interrater agreement was calculated for two transcripts scored by three physicians and proved unacceptably low (average 60\%). Even so, following consultation and refinements to achieve consensus in the error assessment, the remaining transcripts were scored by only one analyst. On that basis, the authors found a total of 396 errors ( $52 \%$ omissions), i.e. a mean rate of 31 interpreter errors per encounter, $63 \%$ of which were deemed to be of potential clinical significance. While the authors reported that errors committed by ad hoc interpreters were significantly more likely to be of clinical significance $(77 \%$ vs $53 \%$ ), the error total was significantly lower for ad hoc interpreters (165) than for the hospital interpreters (231), who had little or no training.

In a subsequent analysis of the transcribed corpus, Laws et al. (2004) applied a different quality evaluation method, using "conversation segments" rather than words as the unit of analysis, and some 20 nominal coding categories correlated with four levels of "translation quality" (good, fair, poor, false/none). Out of the 1211 segments scored for quality of interpretation, two thirds were coded as "poor" $(28.7 \%)$ or "false/none" $(37.3 \%)$. Coding the segments also for interlocutor status, the authors found that the interpreter was speaking as a participant (rather than interpreting) in about $30 \%$ of all segments, and exemplified these instances of "role exchange" (Vasquez \& Javier 1979) with extracts from the transcripts.

In interpreting studies, the concern with errors in earlier studies (e.g. Cokely 1982) has largely given way to a focus on communicative effect (e.g. Cambridge 1999 and Pöchhacker \& Kadric 1999) and discursive strategies, such as "non-renditions" to coordinate the interaction (Wadensjö 1998 and Rosenberg 2002) or the use of direct vs indirect or reported speech (e.g. Dubslaff \& Martinsen 2005), usually in connection with the interpreter's participation status or role in the interaction.

\subsection{Interpreter performance}

Performance in the role of interpreter is at the heart of a large and diverse body of studies generated from various disciplinary perspectives. From the overview in section 1 , role performance emerges as a key topic that has been discussed since the very first publications on interpreting in health 
care. The question of what interpreters do other than "translating", or assuming a non-involved "conduit role", has been addressed in two major ways: one is the discourse-based (and typically qualitative) analysis of interpreter-mediated encounters, as in the "DI paradigm" of interpreting studies (cf. Pöchhacker 2004: 79), which conceptualizes interpreting as coconstructed (dialogic) discourse-based interaction, drawing on a range of sociolinguistic (microsociological, discourse-analytical, conversation-analytical) frameworks (e.g. Merlini \& Favaron 2005; Metzger 1999; Tebble 1999; Valero 2005 and Wadensjö 1998). While typical and well-developed, this line of research is not limited to the disciplinary perspective of interpreting studies, and includes contributions from linguistic, medical as well as social-science perspectives (e.g. Bolden 2000; Davidson 2002; Elderkin-Thompson et al. 2001; Kaufert \& Putsch 1997 and Marcos 1979).

The other major methodological approach to the study of interpreters' performance is survey research aimed at eliciting role perceptions and expectations as well as various other aspects of performance, as seen by service providers, patients or interpreters themselves. Examples include a survey among service providers and a small group of (spoken-language as well as signed-language) interpreters in Vienna (Pöchhacker 2000a) and the large-scale study by Angelelli (2003, 2004a), who administered an instrument measuring interpreters' attitudes to the visibility or nonvisibility of their interpersonal role ("Interpreter's Interpersonal Role Inventory") to some 100 medical interpreters within a larger sample.

Aside from such quantitative studies, interpreters' attitudes, experiences and perceptions regarding their work have been the subject of several (relatively recent) surveys based on qualitative data collected through interviews. Dysart-Gale (2005) conducted semistructured (individual as well as group) interviews with 17 experienced interpreters (for languages including Spanish, Arabic and Russian) practicing in urban hospital settings, and highlighted the inadequacy of the conduit or "transmission model" of interpreting in health care. The study by Hsieh (cf. section 1.6), also based on in-depth interviews, yielded similar findings on role conflicts and strategies for resolving them. Leanza (2005) elicited data on role perceptions from eight pediatrics residents in Switzerland through stimulated-recall interviews based on video-recordings, and in Germany, Allaoui (2005) interviewed five physicians and five hospital interpreters (for Arabic, Dari/Farsi, Bulgarian, Hungarian and Russian) about their respective perceptions of the interpreter's role and task(s). In the more advanced professional context of California, Angelelli (2006) conducted a focus-group study aimed at validating the California Standards for Healthcare Interpreters and found discrepancies between the 'code' and interpreters' actual conduct in challenging situations. More controversially, Green, Free et al. (2005) interviewed 76 bilingual children and youth in the age range of ten to 18 years about their work as interpreters in healthcare settings. While some of the accounts clearly bring out the young mediators' limitations, the authors conceptualize the work of child interpreters not merely as "inappropriate and inadequate interpreting" but as "an oppor- 
tunity and benefit" and "an invaluable contribution to the informal health care economy" (2005: 2109).

Findings that contravene mainstream assumptions about professional community interpreting also emerged from the qualitative study by Edwards et al. (2005), who had research assistants carry out ten semistructured interviews each with members of five ethnic groups in England (Chinese, Kurdish, Bangladeshi, Gujerati, Polish). With special reference to the notion of trust, interviewees expressed a preference for family and friends as interpreters. This idea also emerged from the hospital-based survey by Kuo \& Fagan (1999), who asked 149 Spanish-speaking patients and 51 English-speaking residents about their use of and preferences for different types of interpreters. From differential satisfaction rates of patients $(85 \%)$ and physicians $(61 \%)$ the authors concluded that the use of family members and friends as interpreters should be more seriously considered.

Studies on interpreting and the performance of interpreters from the view of the client or patient are not very numerous. Most surveys of limited-language-proficiency patients, though usually including interpreting as a factor in service provision, are mainly concerned with patient satisfaction in general (e.g. Lee et al. 2002) and with the role of language barriers and various methods of overcoming them (cf. section 2.3) in the delivery of care (cf. section 2.4). Noteworthy examples of evaluation research including the client perspective are the surveys by Mesa (2000) and by Garber \& Mauffette-Leenders (1997). The latter had agency interpreters administer a translated feedback form to Vietnamese, Polish and Portuguese clients (34 respondents) and also elicited assessments from the respective service providers.

\subsection{Communicative practices}

Faced with the need to communicate with patients who do not speak the service providers' language (such as the Deaf as well as migrants of various kinds), healthcare institutions have resorted to various strategies for overcoming linguistic and cultural barriers. Assessing such communication needs and documenting the prevalence of various methods of cross-cultural patient-provider communication is thus a basic concern and has been addressed in numerous (usually questionnaire-based) surveys. Some of these have been targeted at the hospital (management) level (e.g. Bischoff \& Loutan 2004; Carter-Pokras et al. 2004 and Ginsberg et al. 1995) whereas others have targeted service providers themselves in various branches of health care, such as primary care physicians (e.g. Hornberger et al. 1997), outpatient clinicians (e.g. Karliner et al. 2004), mental health professionals (e.g. Drennan 1996 and Minas et al. 1994) and physiotherapists (e.g. Jaggi \& Bithell 1995). In a study covering twelve hospitals in Vienna (Pöchhacker 2000b), data were collected from a total of 508 nurses and therapists as well as doctors in six medical specialties. The picture that emerges from such quantitative survey research, a detailed report of which 
is beyond the scope of this paper, is largely similar: heavy reliance on family members and friends as interpreters; use of bilingual (often nonclinical) staff and in-house interpreters with little or no training; and a sense that a 'professional' interpreting service is preferable or would be desirable but might not be feasible under current economic constraints. In many contexts, the situation appears to be the same as that described by Putsch (1985: 3344) more than twenty years ago: "Institutions vary in their arrangements to meet the needs of monolingual patients and health care providers. Even when there is a well-described need, many facilities have not dealt with language and cultural problems in a formal operational sense."

Addressing communication problems "in a formal operational sense" implies the need for a comparative assessment of various methods for overcoming the language barrier, such as using professional vs ad hoc interpreters, on-site vs remote interpreting (cf. Azarmina \& Wallace 2005 and Bischoff \& Grossmann 2006), or cross-cultural communication without recourse to an intermediary. A number of such studies have been carried out, establishing preferences and perceived advantages from a patient as well as a provider perspective and/or measuring the impact of various interpreting arrangements on such factors as patient satisfaction, cost and length of consultation.

Among the 301 primary care physicians in northern California, for instance, who responded by mail or phone to Hornberger et al.'s (1997) questionnaire, those using trained interpreters $(n=36$, accounting for an estimated $6 \%$ of encounters) rated the quality of interpreting services significantly higher than those using medical staff without interpreter training or family members and friends. However, a multiple regression analysis controlling for demographic and contextual variables yielded no significant differences in quality ratings for these three interpreting arranements. Lee et al. (2002) used a self-administered post-visit questionnaire to survey 303 Spanish-speaking patients in a walk-in clinic in Colorado. An interpreter was used for 175 of these patients, either over the phone $(\mathrm{n}=59)$ or on site (69 family members, 47 ad hoc interpreters). Overall visit satisfaction rates for patients using telephone interpreters were as high as for language-concordant (i.e. Spanish-only) consultations while patients using family or ad hoc interpreters were significantly less satisfied. A similar but interview-based study in a pediatric emergency department in Texas (Garcia et al. 2004) compared 180 parents' satisfaction rates for hospital-trained, ad hoc or telephone interpreters. While the hospital's trained interpreters were judged most favorably, the authors also found a strong correlation between satisfaction with interpreting method and satisfaction with physician, suggesting the presence of additional factors affecting interpreter-related preferences.

In a smaller study carried out in England (Jones et al. 2003), 35 Turkish-speaking patients seeing a general practitioner used either on-site interpreting $(\mathrm{n}=14)$, telephone interpreting $(\mathrm{n}=10)$ or videoconference interpreting $(\mathrm{n}=11)$. Using standardized instruments as well as interviews, 
the authors found comparable patient satisfaction rates for all three methods but also signs of somewhat impaired understanding in videoconferenceinterpreted consultations.

Fagan et al. (2003) studied the impact of various interpreting arrangements (none, hospital interpreter, telephone interpreter or patientsupplied interpreter) on visit length in a Rhode Island outpatient clinic. More than half of the 172 patients using some form of interpreting supplied their own interpreter, and 51 used a hospital interpreter. It was only for the latter that the author did not find significantly different provider and clinic times compared to visits without interpreting. While other interpretermediated consultations were found to take some $15 \%$ to $30 \%$ longer (i.e. an extra 4.4 to 8.3 minutes), hospital interpreters were found to shorten provider times $(-1.2 \mathrm{~min}$.) compared to the average 28 -minute duration of an unmediated consultation. Though the relevance of these findings seems limited in the absence of data on visit content and severity of complaints, they could well be linked up with discourse-based findings regarding hospital interpreters (with or without training) taking on interviewer roles and thus speeding up the consultation (cf. Bolden 2000 and Davidson 1998).

In a more comprehensive and rather unique study, Hornberger et al. (1996) compared communication and interpreting quality as well as participants' satisfaction rates in interpreter-mediated postpartum visits by 49 Spanish-speaking mothers who used either conventional face-to-face interpreting or an experimental remote-simultaneous interpreting system. The latter was found to be associated with higher accuracy rates (in terms of Barik's omissions, additions and substitutions) and a higher number of utterances by physicians and mothers, both of whom - unlike the interpreters - preferred the remote-simultaneous method.

\subsection{Service provision}

Though it is often difficult to distinguish research on particular communicative practices from those on the cross-cultural provision of care, research into the latter thematic orientation focuses not so much on the specifics of interpreting arrangements as such as on their impact on the provision of health care services in general, as measured with regard to service use, clinical outcomes (quality of care), patient satisfaction and costs. Studies in this area constitute the mainstay of research into healthcare interpreting in the medical sciences as documented in the annotated bibliography by Jacobs et al. (2003) and reviewed most recently and thoroughly by Bischoff (2006), Flores (2005) and Jacobs et al. (2006).

Among the most significant contributions on the effect of interpreting services on care delivery is the two-year retrospective cohort study by Jacobs et al. (2001), who compared the receipt of clinical and preventive services by over 4,300 clients of a New England health maintenance organization before and after the introduction of a professional interpreter 
service and found that the 327 patients who used the interpreting service had a significantly greater increase in office visits, prescriptions written and filled, and rectal exams compared to a control group. By the same token, Bernstein et al. (2002) reviewed over 26,000 emergency department records and analyzed a dataset of 500 patients with comparable visit characteristics. Comparing 63 non-English-speaking patients who received professional interpretation to a matched control group of English-speaking patients as well as 374 non-English speakers without interpretation, the authors found that the use of trained interpreters was associated with increased intensity of services, reduced return rates, and lower 30-day charges.

In a similar setting, Baker et al. (1998) surveyed 457 Spanishspeaking patients, of whom 120 used an interpreter (mostly ad hoc) and another 100 communicated directly but said - in a post-visit interview one week later - that an interpreter should have been called. Patient satisfaction with interpersonal aspects of care (i.e. the provider's friendliness, respectfulness, concern, etc., rated as excellent, very good, good, fair or poor) was lower in the interpreter-use group and lowest among those who felt an interpreter should have been used. In this as well as in some other studies, the meaningfulness of empirical findings on the effect of interpreting is limited by a lack of precise definitions and distinctions regarding the interpreters involved. Green, Ngo-Metzger et al. (2005), for instance, mailed an 81-item survey to over 1,300 Chinese and Vietnamese immigrant patients of (eleven) community health centers to elicit their perceptions of communication quality during their most recent visit. Respondents were asked to indicate whether they had used "one of the clinic's interpreters", but no further information on the interpreters - or the clinicians - was available to interpret the findings.

Last, but not least, a major issue associated with the role of interpreting services in the provision of care is cost. Studies devoted to this topic include the survey by Drennan (1996) among 29 clinical staff members in a South African mental hospital, where nurses (67\%) and cleaners (10\%) provided the bulk of 93.5 documented hours of interpreting over a twomonth period; the comprehensive cost-category analysis by Hornberger (1998); the critical review of economic evaluation by Bowen \& Kaufert (2003); the cost-benefit analysis by Jacobs et al. (2004), who estimate the cost of interpreting in their care delivery study (Jacobs et al. 2001) at US\$ 279 per person per year; and the review by $\mathrm{Ku} \&$ Flores (2005), with special reference to insurance and healthcare financing policies.

\subsection{Diversity management policy}

Policy aspects are probably the most 'transdisciplinary' theme in healthcare interpreting research, at least from the vantage point of interpreting studies. Clearly, though, research from the perspectives of law, management and organization is indispensable to understanding - and influencing - the overall development of this field. Aside from the seminal review by 
Woloshin et al. (1995), key contributions on legal and policy issues have been made by Julia Puebla Fortier (e.g. 1997) as well as by Niels AggerGupta (2001) in his grounded-theory study on the implementation of healthcare interpreting services in the United States and Canada.

\section{Methods}

It is quite evident from the overview of research in sections 1 and 2 above that the literature on healthcare interpreting features diverse disciplinary perspectives and methodological approaches. One could indeed speak of a variety of paradigms (in the Kuhnian sense of paradigm as a "disciplinary matrix"), that is, shared worldviews, values, problems, methods and models joining together a given scientific community. In this case, then, there would be several research communities, or paradigms, not only in the crude terms used to structure section 1 but also within such fields as linguistics, sociology and medical science (as well as interpreting studies). And yet, the boundaries between some of these would be hard to define, and many tools and strategies in the methodological repertoire, which often help define a paradigm, are in principle available to researchers across different fields. Looking at the healthcare interpreting literature from yet another angle, I will therefore review it in terms of some basic methodological choices, most of which have already been mentioned in the overview(s) above.

\subsection{Theoretical vs empirical}

The most fundamental choice, which often seems to be taken for granted, is that between theoretical and empirical research. In the context of health care and often in interpreting studies as well, 'research' is generally understood to refer to empirical studies, with little regard for theoretical or conceptual analysis. While the study of interpreting is of course not a purely philosophical matter, the role of theoretical assumptions and conceptual frameworks would seem to warrant more explicit attention. As pointed out with reference to some medical studies (cf. section 2.4), key notions such as 'professional interpreting', 'training', 'accuracy' and 'translation quality' are often ill-defined. Although there are also studies reflecting considerable sophistication (e.g. Lang 1975 and Laws et al. 2004), research into the quality of mediated communication could still benefit from a more socioculturally and context-sensitive function-oriented approach.

One particularly consequential theoretical distinction is that between the 'biomedical' and the 'ethnomedical' model, or paradigm, in the health sciences. Informed by anthropological and cross-cultural research, reflection on the interplay of culture, communication and care (e.g. Kleinman et al. 1978) forms an essential underpinning to the study of such vexing healthcare interpreting issues as the interpreter's role and professional ethics (e.g. Kaufert \& Putsch 1997 and Bischoff in Meyer et al. 2003). 


\subsection{Quantitative vs qualitative}

The role of theory notwithstanding, studying interpreting as a human activity and social phenomenon implies having recourse to empirical data. From the overviews above, it is clear that healthcare interpreting research relies on both quantitative and qualitative data. For studies with a linguistic orientation (cf. section 1.2), it seems natural to treat discourse as qualitative data, taking into account, where possible, not only the verbal but also the nonverbal (paralinguistic and kinesic) dimensions of language. In medical studies, on the other hand, results are generally sought in numerical form so that they can be described statistically and tested for generalizability to the larger population, whereas social-science approaches typically allow for quantitative as well as qualitative data processing. But these categorical distinctions need not hold. On the contrary, quantitative (or even corpuslinguistic) analyses of interpreted medical discourse (e.g. Rosenberg 2002) can be as revealing as clinical comparisons relying (also) on qualitative data (e.g. Jones et al. 2003). Indeed, triangulating various data sources and combining qualitative and quantitative approaches to a given research question (e.g. Leanza 2005) is increasingly regarded as 'best methodological practice' and goes some way toward overcoming the traditional distinction, in some quarters, between 'rigorous quantitative' and less reliable or valid qualitative ('soft science') research.

An illustrative example of the need to combine quantification and qualitative (observational) data can be found in the attempt by Laws et al. (cf. section 3.1) to apply a complex and rigorous evaluation method to the renditions by hospital interpreters and ad hoc interpreters, including an 11year-old sibling. The finding that the young family interpreter achieved the highest translation quality score prompted Laws et al. (2004: 76) to explain: "However, there were many serious problems with this visit, mostly related to interaction processes which our system was not designed to capture." (The physician apparently addressed the girl rather than the mother and ignored the latter's interpreted utterances, many of which included mistranslations of clinical significance). Examples like this highlight the need for researchers to be keenly aware of what can be gained (e.g. in statistical power), and what may be lost, when data from individuals and individual communicative encounters are aggregated for quantification.

\subsection{Evidence vs experience}

In the medical setting, in particular, the distinction between quantitative and qualitative research can also be framed in terms of evidence-based vs experience-based inquiry. In the paradigm of evidence-based medicine, large datasets are used to bring all available clinical knowledge to bear on individual cases, which in turn have to be described in terms of standard indicators of clinical conditions and outcomes (cf. section 2.4). This 
inherently nomothetic approach (i.e. abstracting from individual features to form aggregates) contrasts with the researcher's interest in unique human experience as the prime source of understanding a phenomenon (i.e. the idiographic approach). Apart from self-reports of an interpreter's everyday practice (e.g. Haffner 1992), examples of such work include the qualitative interview-based studies by Edwards et al. (2005) and Green, Free et al. (2005), described in section 2.2.

In the deductive nomothetic orientation, the relevant parameters are predefined by the researcher and applied to the data. This leaves little room for 'other' conceptual categories, like those supplied by patients from other sociocultural backgrounds. Engaging with the latter requires an idiographic or, more specifically, ethnographic approach, seeking to make sense of a phenomenon (such as a case of illness or a communicative interaction) through the eyes and from the perspective of the 'cultural Other'.

Adopting an ethnographic methodology implies respect for and interest in the individual case over and above the desire to generalize. Indeed, case study is an established method in medical research, and the literature on healthcare interpreting includes a number of significant examples. Baxter \& Cheng (1996), for instance, described a case of interpreter-mediated individual psychotherapy (32 sessions) for a 15-yearold Cantonese-speaking girl; Jacobs et al. (1995) presented the case of a ten-year-old Muslim Asian girl suffering a post-traumatic stress reaction after interpreting for her parents in the hospitalization of her terminally ill baby brother; and Parnes \& Westfall (2003) reported on severe anxiety in a Greek geriatric patient in anticipation of a hospital visit involving a professional interpreter rather than her daughter.

Case studies in healthcare interpreting need not be limited to the single case of a particular patient, of course. Conceived as an intensive (usually multimethod) contextualized analysis of an individual unit (person, encounter, intervention, institution, etc.), case-study research seems particularly suited to the study of healthcare interpreting, both at the level of cross-cultural interpersonal interactions and with regard to particular institutional contexts, such as a clinic, hospital ward, healthcare facility, interpreting agency, health maintenance organization or healthcare system. Noteworthy examples of such work include the ethnographic study by Crawford (1994) in three South African day hospitals, and the ethnographic accounts by Davidson (1998) and Angelelli (2004b) based on participant observation of interpreter services in Californian hospitals. As the latter two studies show, the case-study method does not preclude the attempt to draw more general conclusions. On the contrary, the hope is certainly to accumulate case studies with a view to gaining more general insights and identifying relationships that hold across individual cases. This inductive approach, moving from (qualitative) empirical data via content analysis to theoretical insights, also known as grounded theory (as opposed to testing a theory-derived hypothesis against data), informs the work of Agger-Gupta (2001) on the institutional process of establishing healthcare interpreter services and the focus-group study by Ngo-Metzger et al. (2003) on culture- 
specific aspects of healthcare and interpreting quality among Chinese- and Vietnamese-American patients. It remains controversial whether and how researchers can collect and analyze data without bringing their personal experiences and theoretical preconceptions to bear on the process, but this is a general epistemological issue on which those studying healthcare interpreting will have to take sides for themselves.

\subsection{Strategies and techniques}

The picture emerging from the present review of research into healthcare interpreting is undoubtedly one of great methodological diversity. In the face of multiple paradigms and thematic orientations, there is little use in arguing generally for or against particular research strategies and techniques, most of which are open to those in the linguistic, social and medical sciences alike.

Using the broad three-fold distinction of overall research strategies suggested for interpreting studies as a whole (Pöchhacker 2004: 63), that is, fieldwork, survey and experimental research, one can note that none of these approaches is limited to a particular disciplinary domain. Fieldwork, for example, in the prototypical sense, stood at the very beginning of empirical research into healthcare interpreting both in linguistics (Lang 1975) and mental health (Price 1975), and is in a way done by default when clinical practitioner-researchers carry out studies in their field (see, e.g., Drennan \& Swartz 1999). In addition, discourse data are collected 'in the field', by audio- or videorecording, for subsequent qualitative and/or quantitative analysis, whether by researchers in communication and interpreting studies (e.g. Wadensjö 1998) or by medical researchers (e.g. Flores et al. 2003). By the same token, survey research of larger study populations, using on-site or mailed self-administered questionnaires or (face-to-face or over-the-phone) interviews, has been used to good effect in the health and social sciences as well as in interpreting studies, with the unique option in clinical research of surveying a cohort of patients retro- or prospectively on the basis of medical charts and other patient-related information (e.g. billing).

Aside from document analysis of clinical data, which is hardly accessible to those outside the health sciences, medical researchers are also in a unique position to undertake experimental research. Indeed, nonclinical researchers could not normally aspire to the "'gold standard' of a randomized controlled trial" (Jacobs et al. 2006: 124). Nevertheless, various types of before-and-after intervention studies (cf. Bischoff, in this volume) may well be feasible. Such 'lighter' forms of experimentation as simulation and role-play have been used to good effect (e.g. Cambridge 1999 and Cokely 1982), particularly in the context of training and assessment (e.g. Dubslaff \& Martinsen 2005).

There should thus be ample room for those in different domains of healthcare interpreting research to consider complementing their conven- 
tional repertoire with research designs used in other paradigms. For sociolinguists and interpreting scholars this might include quality rating schemes and focus-group techniques as well as ethnographic fieldwork, provided that they manage to gain access to the field. Conversely, interpreting studies should be able to offer social and medical scientists a few insights into cognitive processes and discourse features as well as know-how on matters of assessment, qualifications and training. Such methodological crossfertilization would necessitate a fair amount of interdisciplinary interaction and cooperation, which is difficult to achieve but would ultimately benefit this field of research as a whole.

\section{Conclusion}

Beyond the above conclusions regarding strategies and methods of inquiry, it seems appropriate to stress once again that the field of healthcare interpreting research is characterized first and foremost by its enormous diversity - of paradigms, themes and methodological approaches, not to mention the cultural/ethnic/linguistic diversity of healthcare clients that gives rise to the issue of language barriers and interpreter use in the first place. Even so, the body of literature reviewed here reflects a dominance of US-based medical studies, many of which involve Spanish-speaking patients. Assuming that English-language peer-reviewed journals are equally accessible to authors throughout the world, there seems to be room for many further contributions from diverse sociocultural backgrounds.

If there is one cross-cutting theme in the field under study, it is that of quality - at multiple levels: translation quality, performance quality, quality of communication, quality of care, and quality (equity) in the healthcare system as a whole. Improvements in all these dimensions are seen and sought after as a benefit, but increasingly weighed up against costs. Research concentrated on discourse patterns, quality assessment, performance standards and role expectations, as typically done from an interpreting studies perspective, will therefore not, in and of itself, suffice to demonstrate the 'benefits' of state-of-the-art interpreting arrangements. In order to make an impact on the actual practice of healthcare interpreting, by demonstrating, for instance, which interpreting arrangements are most effective under particular circumstances, and at what cost, human and methodological resources will have to be pooled, maximizing expertise and the reliability and validity of findings. It would be gratifying if this overview of research and methodology of healthcare interpreting could serve the research community for that purpose. 


\section{Bibliography}

Agger-Gupta, Niels (2001). From "Making Do" to Established Service, the Development of Health Care Interpreter Services in Canada and the United States of America. PhD thesis, The Fielding Graduate Institute.

Allaoui, Raoua (2005). Dolmetschen im Krankenhaus. Rollenerwartungen und Rollenverständnisse. Göttingen: Cuvillier.

Angelelli, Claudia (2003). "The interpersonal role of the interpreter in cross-cultural communication". Brunette et al. (eds) (2003), 15-26.

Angelelli, Claudia V. (2004a). Revisiting the Interpreter's Role. A study of conference, court, and medical interpreters in Canada, Mexico, and the United States. Amsterdam/Philadelphia: John Benjamins.

Angelelli, Claudia V. (2004b). Medical Interpreting and Cross-cultural Communication. Cambridge: Cambridge University Press.

Angelelli, Claudia V. (2006). "Validating professional standards and codes: Challenges and opportunities". Interpreting 8(2) (175-193).

Azarmina, P. \& P. Wallace (2005). "Remote interpretation in medical encounters: A systematic review". Journal of Telemedicine and Telecare 11, 140-145.

Baker, David W., Risa Hayes \& Julia Puebla Fortier (1998). "Interpreter use and satisfaction with interpersonal aspects of care for Spanish-speaking patients". Medical Care 36(10), 14611470.

Baxter, Helen \& Louis Yang-ching Cheng (1996). "Use of interpreters in individual psychotherapy". Australian and New Zealand Journal of Psychiatry 30, 153-156.

Bernstein, Judith et al. (2002). "Trained medical interpreters in the emergency department: Effects on services, subsequent charges, and follow-up. Journal of Immigrant Health 4(4), 171176.

Bischoff, Alexander (2006). Caring for migrant and minority patients in European hospitals: A review of effective interventions. Neuchâtel/Basel: Swiss Forum for Migration and Population Studies.

Bischoff, Alexander \& Florian Grosmann (2006). Telefondolmetschen im Spital. Basel: Institut für Pflegewissenschaft, Universität Basel.

Bischoff, Alexander \& Louis Loutan (2004). "Interpreting in Swiss hospitals". Interpreting 6(2), 181-204.

Bloom, Martin et al. (1966). "The use of interpreters in interviewing”. Mental Hygiene 50, 214217.

Bolden, Galina B. (2000). "Toward understanding practices of medical interpreting: Interpreters' involvement in history taking". Discourse Studies 2(4), 387-419.

Bot, Hanneke (2005a). Dialogue Interpreting in Mental Health. Amsterdam/New York: Rodopi.

Bot, Hanneke (2005b). "Dialogue interpreting as a specific case of reported speech". Interpreting $7(2), 237-261$.

Bowen, Sarah \& Joseph M. Kaufert (2003). "Assessing the 'costs' of health interpreter programs: The risks and the promise". Brunette et al. (eds) (2003), 261-272.

Brunette, Louise et al. (eds) (2003). The Critical Link 3: Interpreters in the Community. Amsterdam/Philadelphia: John Benjamins.

Cambridge, Jan (1999). "Information loss in bilingual medical interviews through an untrained interpreter". The Translator 5(2), 201-219.

Carr, Silvana E. et al. (eds) (1997). The Critical Link: Interpreters in the Community. Amsterdam/ Philadelphia: John Benjamins.

Carter-Pokras, Olivia et al. (2004). "Providing linguistically appropriate services to persons with limited English proficiency: A needs and resources investigation". American Journal of Managed Care 10 (Special Issue), SP29-SP36.

Cicourel, Aaron V. (1981). "Language and medicine". C. A. Ferguson \& S. B. Heath (eds) (1981). Language in the USA. Cambridge: Cambridge University Press, 407-429.

Cokely, Dennis (1982). "The interpreted medical interview: It loses something in the translation". The Reflector 3, 5-10.

Crawford, Athalie (1994). Black Patients, White Doctors: Stories lost in translation. Cape Town: National Language Project.

Davidson, Brad (1998). Interpreting Medical Discourse: A study of cross-linguistic communication in the hospital clinic. PhD thesis, Stanford University.

Davidson, Brad (2002). "A model for the construction of conversational common ground in interpreted discourse". Journal of Pragmatics 34, 1273-1300.

Drennan, Gerard (1996). "Counting the cost of language services in psychiatry". South African Medical Journal 86, 343-345. 
Drennan, Gerard \& Leslie Swartz (1999). “A concept over-burdened: Institutional roles for psychiatric interpreters in post-apartheid South Africa". Interpreting 4(2), 169-198.

Dubslaff, Friedel \& Bodil Martinsen (2005). "Exploring untrained interpreters' use of direct versus indirect speech". Interpreting 7(2), 211-236.

Dysart-Gale, Deborah (2005). "Communication models, professionalization, and the work of medical interpreters". Health Communication 17, 91-103.

Ebden, Philip et al. (1988). "The bilingual consultation". Lancet, February 13 (8581), 347.

Edwards, Rosalind, Bogusia Temple \& Claire Alexander (2005). "Users' experiences of interpreters: The critical role of trust". Interpreting 7(1), 77-95.

Elderkin-Thompson, Virginia, Roxane Cohen Silver \& Howard Waitzkin (2001). "When nurses double as interpreters: A study of Spanish-speaking patients in a US primary care setting". Social Science \& Medicine 52, 1343-1358.

Englund Dimitrova, Birgitta et al. (eds) (2006). The Critical Link 4: Interpreters in the Community. Amsterdam/Philadelphia: John Benjamins.

Fagan, Mark J. et al. (2003). "Impact of interpretation method on clinic visit length". Journal of General Internal Medicine 18, 634-638.

Fisher, Sue \& Alexandra D. Todd (eds) (1983). The Social Organization of Doctor-Patient Communication. Washington, DC: Center for Applied Linguistics.

Flores, Glenn (2005). "The impact of medical interpreter services on the quality of health care: A systematic review". Medical Care Research and Review 62(3), 255-299.

Flores, Glenn et al. (2003). "Errors in medical interpretation and their potential clinical consequences in pediatric encounters". Pediatrics 111(1), 6-14.

Garber, Nathan \& Louise A. Mauffette-Leenders (1997). "Obtaining feedback from non-English speakers". Carr et al. (eds) (1997), 131-143.

Garcia, E. A. et al. (2004). "A comparison of the influence of hospital-trained, ad hoc, and telephone interpreters on perceived satisfaction of limited English-proficient parents presenting to a pediatric emergency department". Pediatric Emergency Care 20(6), 373-378.

Ginsberg, C. et al. (1995). Interpretation and Translation Services in Health Care: A survey of US public and private teaching hospitals. New York: National Public Health and Hospital Institute.

Green, Alexander R., Quyen Ngo-Metzger et al. (2005). "Interpreter services, language concordance, and health care quality: Experiences of Asian Americans with limited English proficiency". Journal of General Internal Medicine 20, 1050-1056.

Green, Judith, Caroline Free et al. (2005). "Translators and mediators: Bilingual young people's accounts of their interpreting work in health care". Social Science \& Medicine 60, 20972110.

Haffner, Linda (1992). "Translation is not enough: Interpreting in a medical setting". Western Journal of Medicine 157, 255-259.

Holzmann, Thomas H. et al. (1994). "Ausländische Patienten in stationärer Behandlung in einer psychiatrischen Universitätsklinik mit Versorgungsauftrag”. Psychiatrische Praxis 21, 106-108.

Hornberger, John (1998). "Evaluating the costs of bridging language barriers in health care". Journal of Health Care for the Poor and Underserved 9(Supplemental), S26-S39.

Hornberger, John, Haruka Itakura \& Sandra R. Wilson (1997). "Bridging language and cultural barriers between physicians and patients". Public Health Reports 112, 410-417.

Hornberger, John C. et al. (1996). "Eliminating language barriers for non-English-speaking patients". Medical Care 34, 845-856.

Hsieh, Elaine (2006). "Conflicts in how interpreters manage their roles in provider-patient interactions". Social Science \& Medicine 62, 721-730.

Jacobs, B. et al. (1995). "The hazards of using a child as an interpreter". Journal of the Royal Society of Medicine $88,474 \mathrm{P}-475 \mathrm{P}$.

Jacobs, Elizabeth et al. (2001). "Impact of interpreter services on delivery of health care to limitedEnglish-proficient patients". Journal of General Internal Medicine 16, 468-474.

Jacobs, Elizabeth et al. (2003). Language Barriers in Health Care Settings: An annotated bibliography of the research literature. Woodland Hills, CA: The California Endowment.

Jacobs, Elizabeth et al. (2004). "Overcoming language barriers in health care: Costs and benefits of interpreter services". American Journal of Public Health 94(5), 866-869.

Jacobs, Elizabeth et al. (2006). "The need for more research on language barriers in health care: A proposed research agenda”. Milbank Quarterly 84(1), 111-133.

Jaggi, Anju \& Christine Bithell (1995). "Relationships between physiotherapists' level of contact, cultural awareness and communication with Bangladeshi patients in two health authorities". Physiotherapy 81(6), 330-337. 
Jones, D. et al. (2003). "An exploratory study of language interpretation services provided by videoconferencing”. Journal of Telemedicine and Telecare 9, 51-56.

Karliner, Leah S., Eliseo J. Pérez-Stable \& Ginny Gildengorin (2004). "The language divide: The importance of training in the use of interpreters for outpatient practice". Journal of General Internal Medicine 19, 175-183.

Kaufert, Joseph M. \& William W. Koolage (1984). "Role conflict among 'culture brokers': The experience of native Canadian medical interpreters". Social Science \& Medicine 18(3), 283-286.

Kaufert, Joseph M. \& Robert W. Putsch (1997). "Communication through interpreters in healthcare: Ethical dilemmas arising from differences in class, culture, language, and power". Journal of Clinical Ethics 8(1), 71-87.

Kleinman, Arthur, Leon Eisenberg \& Byron Good (1978). "Culture, illness, and care: Clinical lessons from anthropologic and cross-cultural research". Annals of Internal Medicine 88, 251-258.

$\mathrm{Ku}$, Leighton \& Glenn Flores (2005). "Pay now or pay later: Providing interpreter services in health care". Health Affairs 24(2), 435-443.

Kuo, David \& Mark J. Fagan (1999). "Satisfaction with methods of Spanish interpretation in an ambulatory care clinic". Journal of General Internal Medicine 14, 547-550.

Lang, Ranier (1975). "Orderlies as interpreters in Papua New Guinea". Papua New Guinea Medical Journal 18(3), 172-177.

Launer, John (1978). "Taking medical histories through interpreters: Practice in a Nigerian outpatient department". British Medical Journal 277(6142), 934-935.

Laws, M. Barton et al. (2004). "A new method for evaluating the quality of medical interpretation". Medical Care 42, 71-80.

Leanza, Yvan (2005). "Roles of community interpreters in pediatrics as seen by interpreters, physicians and researchers". Interpreting 7(2), 167-192.

Lee, Linda J. et al. (2002). "Effect of Spanish interpretation method on patient satisfaction in an urban walk-in clinic". Journal of General Internal Medicine 17, 641-646.

Leininger, M. Madeleine (1997). Culture Care Diversity \& Universality: A Theory of Nursing. New York: National League for Nursing Press.

Leyer, Emanuela Maria (1990). "Verborgene Strukturen in der ärztlichen und psychotherapeutischen Interaktion mit ausländischen Patienten - dargestellt und diskutiert am Beispiel eines türkischen chronisch Schmerzkranken”. Psychotherapie, Psychosomatik, Medizinische Psychologie 40, 423-431.

Marcos, Luis R. (1979). "Effects of interpreters on the evaluation of psychopathology in nonEnglish-speaking patients". American Journal of Psychiatry 136(2), 171-174.

Merlini, Raffaela \& Roberta Favaron (2005). "Examining the 'voice of interpreting' in speech pathology". Interpreting 7(2), 263-302.

Mesa, Anne-Marie (2000). "The cultural interpreter: An appreciated professional". Roberts et al. (eds) (2000), 67-79.

Metzger, Melanie (1999). Sign Language Interpreting: Deconstructing the Myth of Neutrality. Washington, DC: Gallaudet University Press.

Meyer, Bernd (2002). "Medical interpreting: Some salient features". G. Garzone \& M. Viezzi (eds) (2002). Interpreting in the 21st Century. Amsterdam/Philadelphia: John Benjamins, 159169.

Meyer, Bernd et al. (2003). "Analysing interpreted doctor-patient communication from the perspectives of linguistics, interpreting studies and health sciences". Brunette et al. (eds) (2003), 67-79.

Minas, I. H., G. W. Stuart \& S. Klimidis (1994). "Language, culture and psychiatric services: A survey of Victorian clinical staff". Australian and New Zealand Journal of Psychiatry 28, 250-258.

Ngo-Metzer, Quyen et al. (2003). "Linguistic and cultural barriers to care: Perspectives of Chinese and Vietnamese immigrants". Journal of General Internal Medicine 18, 44-52.

Ong, L. M. L. et al. (1995). "Doctor-patient communication: A review of the literature". Social Science \& Medicine 40(7), 903-918.

Parnes, Bennett L. \& John M. Westfall (2003). "An elderly woman with severe anxiety associated with anticipated use of an interpreter". Journal of the American Board of Family Practice $16(3), 255-256$.

Pöchhacker, Franz (2000a). "The community interpreter's task: Self-perception and provider views". Roberts et al. (eds) (2000), 49-65.

Pöchhacker, Franz (2000b). "Language barriers in Vienna hospitals". Ethnicity \& Health 5(2), $113-$ 119.

Pöchhacker, Franz (2004). Introducing Interpreting Studies. London/New York: Routledge. 
Pöchhacker, Franz \& Mira Kadric (1999). "The hospital cleaner as healthcare interpreter: A case study". The Translator 5(2), 161-178.

Porter, Anne (1999). "Sign-language interpretation in psychotherapy with deaf patients". American Journal of Psychotherapy 53(2), 163-176.

Price, J. (1975). "Foreign language interpreting in psychiatric practice". Australian and New Zealand Journal of Psychiatry 9, 263-267.

Prince, Cynthia D. (1986). Hablando con el doctor: Communication problems between doctors and their Spanish-speaking patients. $\mathrm{PhD}$ thesis, Stanford University.

Puebla Fortier, Julia (1997). "Interpreting for health in the United States". Carr et al. (eds) (1997), 165-177.

Putsch, Robert W. (1985). "Cross-cultural communication: The special case of interpreters in health care". Journal of the American Medical Association 254(23), 3344-3348.

Richie, J. (1964). Using an interpreter effectively. Nursing News 12, 27-29.

Roberts, Roda P. et al. (eds) (2000). The Critical Link 2: Interpreters in the Community. Amsterdam/Philadelphia: John Benjamins.

Rosenberg, Brett Allen (2002). "A quantitative discourse analysis of community interpreting". Translation: New Ideas for a New Century. Proceedings of the XVI FIT Congress. Paris: FIT, 222-226.

Sarangi, Srikant (2004). "Editorial: Towards a communicative mentality in medical and healthcare". Communication \& Medicine 1(1), 1-11.

Tebble, Helen (1999). "The tenor of consultant physicians: Implications for medical interpreting". The Translator 5(2), 179-200.

Tomassini, Elena \& Francesca Nicolini (2005). "Survey on the role of community interpreters and cultural mediators in hospitals in the Emilia Romagna region". C. Valero Garcés (ed.) (2005). Translation as Mediation or How to Bridge Linguistic and Cultural Gaps. CD. Alcalá: Universidad de Alcalá, 100-107.

Tribe, Rachel (1999). "Using interpreters/bicultural workers when working with refugee clients, many who have been tortured". British Journal of Medical Psychology 72, 567-576.

Valero Garcés, Carmen (2005). "Doctor-patient consultations in dyadic and triadic exchanges". Interpreting 7(2), 193-210.

Vasquez, Carmen \& Rafael A. Javier (1991). "The problem with interpreters: Communicating with Spanish-speaking patients". Hospital and Community Psychiatry 42(2), 163-165.

Wadensjö, Cecilia (1998). Interpreting as Interaction. London/New York: Longman.

Westermeyer, Joseph (1990). "Working with an interpreter in psychiatric assessment and treatment". Journal of Nervous and Mental Disease 178(12), 745-749.

Woloshin, Steven et al. (1995). "Language barriers in medicine in the United States". Journal of the American Medical Association 273(9), 724-728.

\footnotetext{
1 Language barriers obviously affect - and limit - also the present effort at surveying the literature. A good number of country-specific research initiatives and reports can be assumed to exist, but given the bias toward English in the medical literature, these will generally remain unseen.

2 I am grateful to Alexander Bischoff, Niels Agger-Gupta and Natividad Herrero Prado for helping me access a number of recent reports and papers published in medical journals.

3 Cf. the conferences organized by EACH, the European Association for Communication in Healthcare, in 2002, 2004 and 2006.
} 